\title{
DESIGN OF A FUZZY FRACTIONAL ORDER ADAPTIVE IMPEDANCE CONTROLLER WITH INTEGER ORDER APPROXIMATION FOR STABLE ROBOTIC CONTACT FORCE TRACKING IN UNCERTAIN ENVIRONMENT
}

\author{
Hongli CAO* ${ }^{\oplus}$ \\ * Key Laboratory of Advanced Transducers and Intelligent Control System of Ministry of Education, \\ Taiyuan University of Technology, Taiyuan 030024, China \\ caohongli@tyut.edu.cn
}

received 30 June 2021, revised 17 September 2021, accepted 25 September 2021

\begin{abstract}
Current research in robot compliance control is unable to take both transient contact force overshoots and steady-state force tracking problems into account. To address this problem, we propose a fuzzy fractional order (FO) adaptive impedance controller to avoid the force overshoots in the contact stage while keeping force error in the dynamic tracking stage, where traditional control algorithms are not competent. A percentage gain is adopted to map FO parameters to integer order (IO) parameters by their natural properties, and a fuzzy logical controller is introduced to improve the system stability. The simulation results indicate that the proposed controller can be made more stable than and superior to the general impedance controller, and the force tracking results also have been compared with the previous control methods.
\end{abstract}

Key words: adaptive impedance control, contact interactions, fractional order, fuzzy logic, integer order approximation

\section{INTRODUCTION}

With the development of robot technology, establishing the contact between the robot's end-effector and the environment is becoming a vital part of tasks in robotic applications that involve assembly [1], precise handling or surface processing [e.g., polishing] [2], exoskeleton [3] etc. The main issue in contact establishment concerns the contact force magnitude that may take prohibiting values, which may directly lead to the failure of a task, with even more serious consequences [manipulated object or manipulator itself damaged] at the instant of contact. This force overshoot could be due to delays in measurement or communication, uncertainties regarding the robot/environment model and discontinuities in controller input. To solve this knotty problem, some compliance control methods are proposed by researchers [4].

Hybrid position/force control and impedance control are two main compliance control approaches which can be distinguished by the relationship between positions and force, which are usually used to solve the contact problem. Hybrid position/force control is deter-mined based on formal models of the manipulator and task geometry to divide the task space into two separate subspecies: the position and the force subspaces as proposed by Robert [5, 6 ]. Different control laws are responsible for position control in the free space and for force control along the directions in which position is constrained. The second strategy is an impedance control which describes the relation between force and position, first introduced by Hogan [7]. In this method, neither the position nor the force is used for control, but a generalised impedance equation, which defines the target impedance between the motion and the interaction force/torque, is utilised. In many cases, impedance control outperformed the hybrid position/force control in terms of controlling the dynamic contact between manipulators and the environment, as well as showing more robustness in an unknown stiffness environment [8]. To cope with the need for more complex tasks in practical robotic applications, a variety of studies have combined the concept of the hybrid force/position control (intuitive and easy implementation) and the impedance control (superior interactivity) scheme to form the hybrid impedance control [9-11]. Furthermore, due to the various uncertainties in the robot manipulator and the environment, adaptive hybrid impedance control was required to be a new stable force tracking impedance control scheme, capable of both tracking a desired force and of compensating for uncertainties in environment location and stiffness [12-14].

But at the same time, some advanced nonlinear control schemes are also applied to handle the uncertainties for complex tasks, such as robust impedance control [3], adaptive fuzzy control [15] and neural net-works control [16]. Moreover, to manage the application of industrial robots, the position-based impedance control was proposed, and the performance and stability were also analysed [17, 18]. Among these methods, fuzzy logic brings advanced and useful properties for the robust command of uncertain dynamic systems, which are prone to appearing in practical industrial robot applications, since it allows to interpret and combine several different actions of control by means of linguistic rules and taking advantage of the user experience. These outstanding properties of fuzzy control systems have been considered for designing robust controllers and precise motion planning schemes in the case of robotic plants [19,20].

In addition to using integer order (IO) system models and methods to control robots, some researchers have also turned their eyes to other nonlinear control strategies - namely fractional order (FO) tools - due to the virtue of their inherent properties of 
memory and heritage. From here, the impedance controller has 10 and FO control distinction. The FO controller is widely used to enhance the closed-loop performance by improving trajectory tacking and transient and steady-state responses, and it guarantees the better control performance for both $\mathrm{IO}$ and FO systems [21]. Hence, the FO controller has been widely combined with advanced control techniques such as Proportion Integral Differential (PID) control, optimal control, adaptive control, (Sliding Mode Control) SMC etc. [22-24]. It also has been demonstrated that the FO controller is compatible with useful techniques for the modelling and control of advanced complex systems [25, 26], such as industrial automation control [27] and robotic applications [28-31], but then the problem of calculating the FO needs to be tackled.

Therefore, there are many methods proposed using the integer order approximation (IOA) to solve the FO derivative and integrator operators. However, it is quite difficult to determine the best among these methods due to the performance evaluation criteria having different emphases; examples for this phenomenon include the IOA effect, accuracy of the frequency response and accuracy of the time response applying only to certain situations. In the frequency domain, Oustaloup presents an approximation method based on the recursive distribution of poles and zeros in a limited frequency range to obtain an 10 transfer function by using frequency response fitting [32], which is by far the most common method. Either way, it is still complex, and a reasonable balance needs to be struck between the practicality of robotic industrial applications and their calculation complexity. Fuzzy logic and FO techniques have been considered together for out-standing control applications, the aim being to improve the performance of classical schemes [33].

The above control schemes usually only consider one aspect of the force control problem; in actual robot applications, it is a very intractable problem to both maintain accurate force tracking and refrain from damaging the operating objects. Satisfying three indicators of force control: (1) maintaining force tracking error; (2) avoiding force overshoots; and (3) achieving fast response is still an open problem. Few researchers have considered all the indicators together, most confining themselves to only one aspect.

\subsection{Contribution}

In view of this, the contribution of this paper lies in the validation of the proposed controller, which uses fuzzy logic, FO tools and adaptive control to form a fuzzy FO adaptive impedance control (Fuzzy-FO-AIC) for dynamic force contact control in uncertain environments. The main goal of such a controller is to avoid the force overshoots in the contact stage while keeping force error in the dynamic tracking stage, where traditional control algorithms are not competent. The control scheme does not rely on the FO calculus; thus, a percentage control is adopted to convert it to IO calculations using its natural properties. This approximation method is simpler and more efficient than other IOA methods for robot compliance control problems. More-over, the Fuzzy-FO-AIC is presented here mainly in order to cater to a time-varying environment by adjusting the general impedance controller parameters using a fuzzy controller to achieve a dynamic update rate; its adaptability to time-varying dynamic environments is far superior to traditional impedance control. The stability and robustness of Fuzzy-FO-AIC and its effect on force overshoot suppression and force tracking performance during contact interactions with linear and nonlinear uncertain environments are investigated and com- pared to previous impedance controls. It is expected that it would have a very significant impact on the film precision processing field of industrial robotic applications.

\subsection{Outline}

The paper is structured as follows. Section 'Preliminaries' reviews some preliminaries, control architecture and the problem. The Fuzzy-FO-AIC control scheme is proposed to solve the problem in Section 'Control Architecture'. Section 'Simulations' presents the simulation results to verify the superiority of the proposed control algorithm. Conclusions are presented in Section 'Conclusions'.

\section{PRELIMINARIES}

\subsection{FO system}

FO calculus, a generalisation of well-known IO calculus, allows differentiation and integration of arbitrary orders. Calculating a FO derivative/integral (differ-integral) is not trivial. In multiple mathematically well-founded definitions, one needs to choose the appropriate definition based on the application area. Among them, the Grunwald-Letnikov definition is usually used for calculating the FO. The Grunwald-Letnikov definition for FO operation is given as:

$$
D^{\alpha} y(t)=\lim _{h \rightarrow 0} \frac{1}{h^{\alpha}} \sum_{i=0}^{\frac{t-a}{h}} C_{i}^{\alpha} y(t-i h)
$$

where $\alpha$ is the order, $h$ is the step size of the calculation, a is the window length and $C_{i}^{\alpha}$ can be calculated by following:

$\mathrm{C}_{0}^{\alpha}=1, \mathrm{C}_{\mathrm{i}}^{\alpha}=\mathrm{C}_{\mathrm{i}-1}^{\alpha}\left(1-\frac{\alpha+1}{\mathrm{i}}\right), \mathrm{i}=1,2, \ldots$

FO differ-integral is a linear operator, and it has a memory and relies on the whole history. In real-time implementation, it relies upon the short memory principle due to the forgetting factor. Therefore, the memory storage and calculation amount will be large, which will lead to some obstacles to practical application.

\subsection{Contact control problem}

For modelling the contact between robot and environment, we can consider one of the degrees of the robot system in which a mass interacts with a stiffness environment (Fig. 1). The contact procedure can be divided into three processes: (1) Free motion; (2) Instant contact; (3) Steady tracking. The corresponding contact force curves are shown in Fig. 1(b). It can be seen that a large force overshoot is produced in the collision stage. In the actual robotic application, if not controlled, too much oscillation and force overshoot will cause the system to be unstable and even worse consequences.

In addition to the above requirements, a superior force controller adapting to a variety of complex tasks should have the following characteristics: (1) Maintaining a high accuracy position tracking capability in Free-space (basic capabilities of modern industrial robots); (2) High speed, stable, smooth contact with the collision stage; (3) High precision force tracking capability in Steadycontact. In this study, the design of robot controller is mainly 
aimed at the indicators of (2) and (3). As (1) is a basic capability of industrial robots and the impedance control strategy is built on (1), the following section gives a brief introduction.
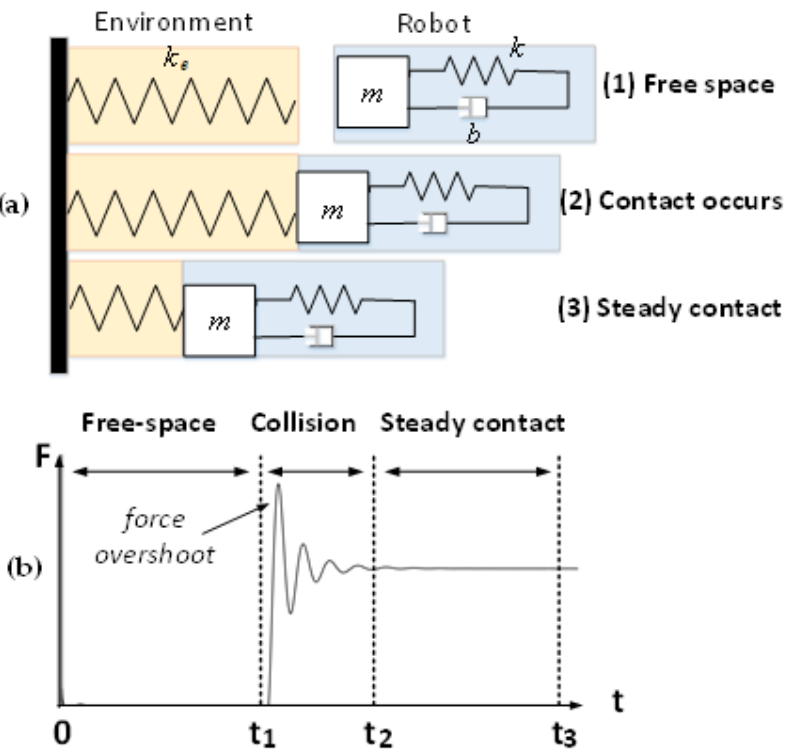

Fig. 1. Contact model of robot and environment

\subsection{Position controlled robot}

For position-based impedance control (also called admittance control in some researches) systems, hierarchical separation of position control and impedance control is a common structure nowadays (Fig. 2). The computed-torque method combined with a conventional proportional-differential (PD) controller can form a superior position controller, in which the actual end-effector's position is equal to that commanded, with no tracking delay and error. Once such a position controller is available, the impedance controller can be built on it.

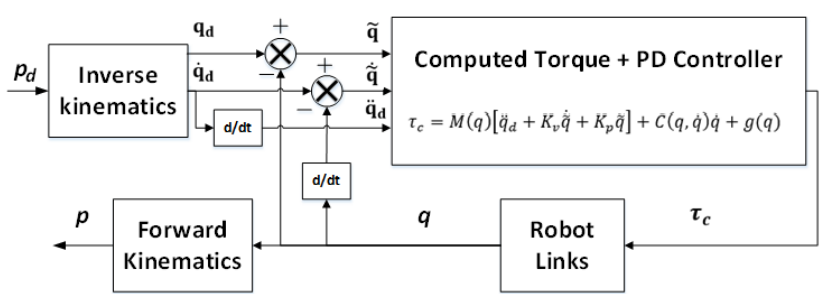

Fig. 2. Generic position controller that is applied to a robot manipulator (computed-torque + PD). PD, proportional-differential

\section{CONTROL ARCHITECTURE}

Fig. 3 shows the whole Fuzzy-FO-AIC control architecture. Firstly, the motion space and constraint space are planned according to the robot's task definition. The robot is required to move its own end-effector with a desired position $P_{d}$, and interact with the environment. The generated interaction force $F_{e}$ between robot and environment is measured by a force sensor, which is attached to the end-effector of the robot. The force error between the desired force $F_{d}$ and the interaction force $F_{e}$ is then sent to the Fuzzy-FO-AIC to generate the corresponding position error e. Then, the robot's servo motion controller transmits sufficient torque to its joints in order to closely achieve the reference position.

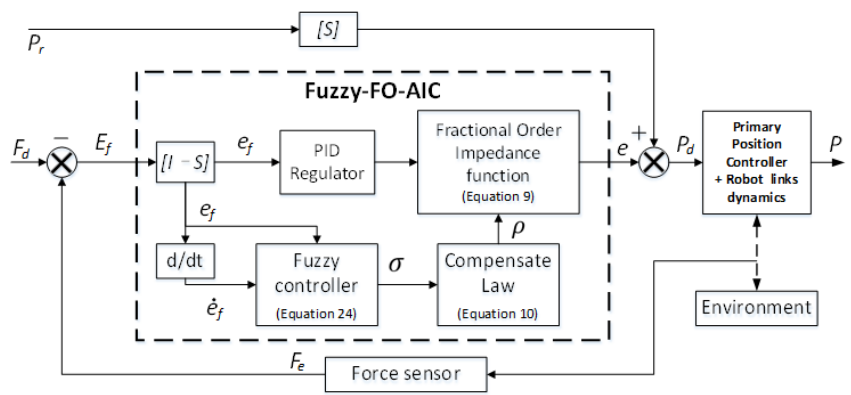

Fig. 3. Fuzzy FO adaptive hybrid impedance control architecture. FO, fractional order

\subsection{Fractional order-adaptive impedance control (FO-AIC)}

\subsubsection{FO-AIC design}

Inspired by the integer order adaptive impedance controller (IO-AIC) that is most commonly used in researches, the difference between FO controller and $\mathrm{IO}$ controller is that the 2-order term in IO-AIC is not an integer but a decimal. Hereby, FO-AIC is defined as:

$$
\begin{aligned}
& Y(s)=\frac{1}{Z_{F O-A I C}(s)}=\frac{1}{m_{d} s^{\alpha}+b_{d}(s+\rho(s))} \\
& \rho(s)=-\frac{\sigma}{b} e_{f}(s)
\end{aligned}
$$

The range of $\alpha$ is defined as $1<\alpha<2$. Here, $m_{d}$ and $b_{d}$ are the desired parameters of impedance controller. Eq. (4) defines the adaptive compensation law; $\rho$ is the damping compensation which is adjusted according to the force sensor information online. From an extreme point of view, for $\alpha=1$, the controller acts like a pure damper due to the order being reduced to 1-order. On the other hand, when the integration order is at $\alpha=2$, it becomes IO-AIC. Therefore, the effective mass and damping supplied by FO-AIC changes depending on the integration order $\alpha$, while disruptive effects are present throughout the range. An important nature property of FO is, as $\alpha$ is decreased gradually from 2 to 1 , the inertial energy storage characteristics of decay and energy dissipation characteristics dominate. To demonstrate the effect of $\alpha$ on the dynamical response of the FO-AIC, considering the frequency domain expression using the Euler transform, we obtain:

$Z=m_{d} \omega^{\alpha} \cos \left(\frac{\alpha \pi}{2}\right)+b_{d}(j \omega+\rho(j \omega))+j m_{d} \omega^{\alpha} \sin \left(\frac{\alpha \pi}{2}\right)$

In Eq. (5), the effective mass and damping provided by FOAIC are $m_{d} \omega^{\alpha} \sin (\alpha \pi / 2)$ and $m_{d} \omega^{\alpha} \cos (\alpha \pi / 2)+$ $b_{d}(j \omega+\rho(j \omega))$, respectively, whereas these values are constant as $m_{d}$ and $b_{d}$ for IO-AIC. Hence, unlike in the IO case, the effective damping in FO-AIC is frequency-dependent and can be adjusted by altering the integration order $\alpha$. This property of FOAIC is of interest for robot force control tasks, as it can improve stability robustness of interactions in tasks involving contacts with 
environment, as can be seen in Fig. 4. From the point of view of controllability, compared with IO-AIC, one more adjustable parameter can ensure the system stability using FO-AIC.

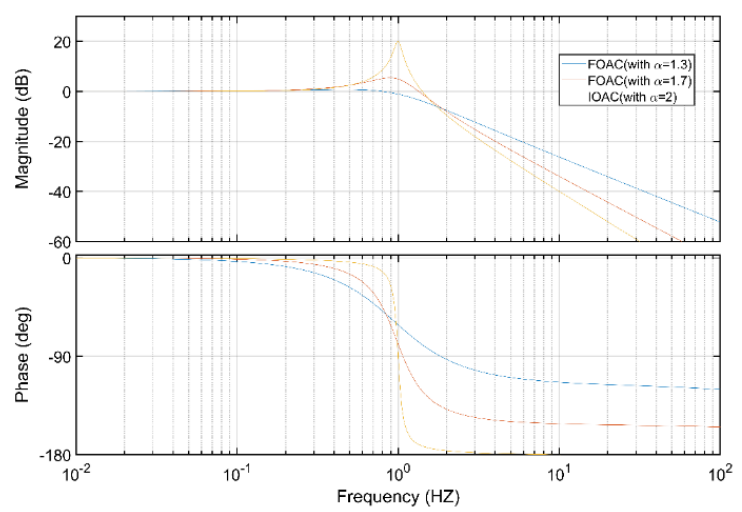

Fig. 4. Bode diagram analysis of FO-AIC and IO-AIC. FO-AIC, fractional order-adaptive impedance control; IO-AIC, integer order adaptive impedance controller

\subsubsection{FO-AIC with IOA}

From the natural property analysis of FO-AIC, we ascertain that the effective mass and damping are frequency-dependent, and can be adjusted by varying the integration order $\alpha$. Based on this property, a natural idea was born - when taking two limits of FO, the FO-AIC becomes IO-AIC $(\alpha=2)$ and a pure damper controller $(\alpha=1)$, respectively. Hence, the new approximation controller can be formed by moving $\alpha$ from the order to the coefficient $\beta(\beta=\alpha-1)$. The coefficient $\beta(0<\beta<1)$ connects the two IO controllers (IO-AIC and a pure damper) by controlling percentages of the output, which does the same thing as $\alpha$ :

$Y(s)=\frac{1}{Z_{F O-A I C}(s)}=\frac{\beta}{m_{d} s^{2}+b_{d}(s+\rho(s))}+\frac{(1-\beta)}{b_{d}(s+\rho(s))}$
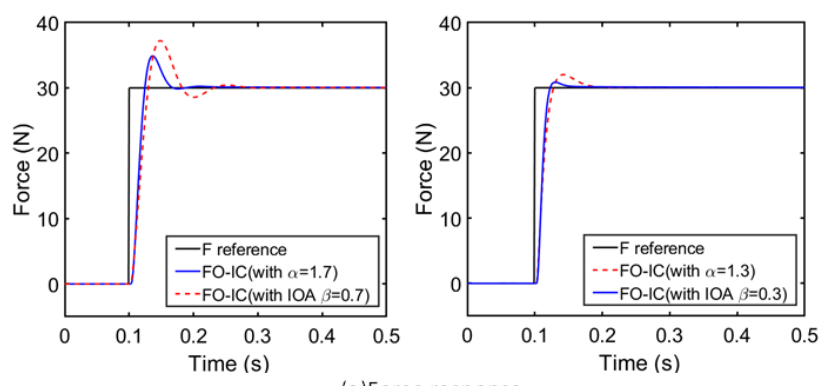

(a)Force response
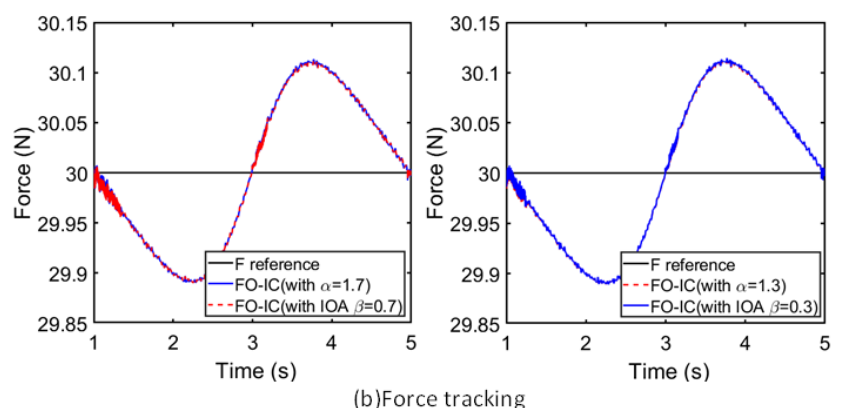

Fig. 5. IOA Effects of FO-AIC. FO-AIC, fractional order-adaptive impedance control; IOA, integer order approximation
From Eq. (3), it can be found that $\beta$ is directly related to the mass and damping effect that the controller wants to present. Similar to contact tasks, it can improve stability and robustness by decreasing $\beta$ in the collision stage (presents its damping characteristics) and increasing $\beta$ in the steady force tracking stage (presents its compensation characteristics), when the robot contacts the environment.

Fig. 5 shows the IOA effects of FO-AIC. It can be seen that there is a certain error in the contact stage between the FO-AIC (with Oustaloup method) and FO-AIC with IOA; due to the fact that FO has a memory and relies on the whole history, the memory effect is removed and replaced by a percentage in FOAIC with IOA. Nevertheless, considering the ease and analyzability of use, this error can be ignored. This means the new IOA methods can be used in robot force control.

\subsection{Pre-PID regulator}

To achieve the force tracking response speed, a pre-PID regulator is designed before FO-AIC, each part forming a PID-like FO-AIC to improve the force tracking performance, which can be expressed as:

$\left(k_{p}+\frac{k_{i}}{s}+k_{d} s\right) e_{f}(s)=\frac{m_{d} s^{2}+b_{d}(s+\rho(s))}{\beta}+\frac{b_{d}(s+\rho(s))}{1-\beta}$

where $k_{p}, k_{i}$ and $k_{d}$ are the positive gains of the pre-PID. Marking $\gamma=k_{p}+k_{i} / s+k_{d} s$, we obtain:

$e_{f}(s)=\frac{m_{d}}{\gamma \beta} s^{2}+\frac{b_{d}}{\gamma \beta(1-\beta)}(s+\rho)$

Compared with the general FO-AIC, it could be found that the desired mass and damp coefficient of the PID-like FO-AIC changes from $m_{d} / \beta$ to $\mathrm{m}_{\mathrm{d}} / \gamma \beta$ and $\mathrm{b}_{\mathrm{d}} / \beta(1-\beta)$ to $b_{d} /(\gamma \beta$ (1$\beta)$ ), respectively. It means that the pre-PID parameter $\gamma$ selection and $\beta$ will affect the response speed and stability of the controller. To make this point clearer, we observe the response behavior of a second-order system by changing its parameters, as shown in Fig. 6.

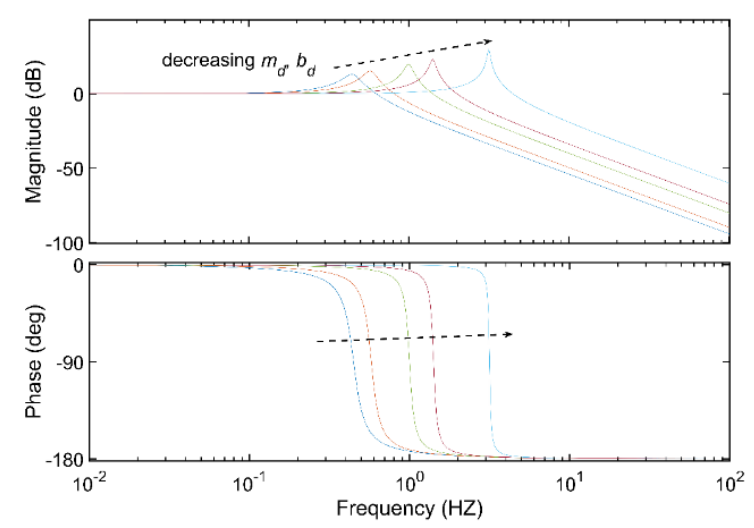

Fig. 6. Frequency response diagrams for decrease of inertia and damping

The selection of $\beta$ has a great impact on the response of the system due to fact that it relates to the nature property of the system. Here, if we were to keep the coefficient $\beta$ a constant value, and the impedance parameters are decreased simultaneously, then both the resonance amplitude and the resonant fre- 
quency are increased. This indicates that if the pre-PID parameters are chosen reasonably, it can achieve a better expectation than the general controller, so that the contact force generated at the end-effector can quickly converge to the desired value.

\subsection{Fuzzy logic design}

It can be seen from Eq. (8) that the response of the FO-AIC is related not only to coefficient $\beta$ and the pre-PID regulator $\gamma$ but also to the compensation rate $\rho$. It is assumed that the position controller for the industrial robot has a high-bandwidth servo loop, and the model uncertainties (inertia, friction and Coriolis etc.) and some external disturbances can be suppressed by PD control. Therefore, the block for the primary position controller with robotlink dynamics in Fig. 2 is dropped; thus, $P_{d} \cong P$. Hence, the robot motion controller can be simplified to gain 1 . The force sensor can be reduced to a linear stiffness environmental model. The whole of the robot control strategy is simplified in Fig. 7.

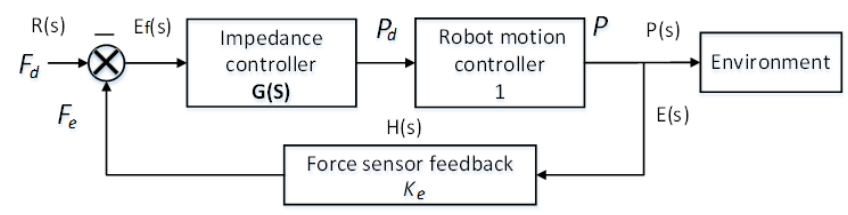

Fig. 7. The transfer function of the simple interaction model

The complete pre-PID, such as FO-AIC with IO approximation and the new design adaptive compensation rate in the time domain, can be represented as:

$e_{f}(t)=\frac{m_{d}}{\gamma \beta} \ddot{\hat{e}}(t)+\frac{b_{d}}{\gamma \beta(1-\beta)}(\dot{\hat{e}}(t)+\rho(t))$

$\rho(t)=\rho(t-T)+\sigma \gamma \beta(1-\beta) \frac{\left(f_{d}(t-T)-f_{e}(t-T)\right)}{b_{d}}$

Given that $\hat{p}_{e}=p_{e}-\delta p_{e}$ is the estimation of the environment location, the estimation position error can then be expressed as $\hat{e}=e+\delta p_{e} \cdot \gamma$ is the pre-PID regulator, and since we're just taking the proportional part here, $\gamma=k_{p}$ is equal to a constant. The initial conditions are given by $\rho(0)=0$, and $T$ is the sampling period (usually smaller is better). The update rate $\sigma$ selection is associated with system stability and performance, which has been analysed in the forthcoming section, and it is used for designing the fuzzy logic controller.

\subsubsection{Transient and steady-state analysis}

According to the principle of dispersion, rewriting Eq. (10) and marking $c(t)=-e_{f}=f_{d}(t)-f_{e}(t)$, n elements of the $\rho$ series can be expanded as: $\frac{b_{d}}{\gamma \beta(1-\beta)} \rho(t)=\frac{b_{d}}{\gamma \beta(1-\beta)} \rho(t-n T)+\sigma c(t-n T)+\cdots+$
$\sigma c(t-T)$

Substituting Eq. (11) into Eq. (9) yields:

$e_{f}(t)=\frac{m_{d}}{\gamma \beta} \ddot{\hat{e}}(t)+\frac{b_{d}}{\gamma \beta(1-\beta)} \dot{\hat{e}}(t)+\sigma(c(t-n T)+\cdots+$

$c(t-T))$
Taking the Laplace transform of Eq. (12),

$E_{f}(s)=\left(\frac{m_{d}}{\gamma \beta} s^{2}+\frac{b_{d}}{\gamma \beta(1-\beta)} s\right) \hat{E}(s)+\sigma\left(e^{-n T s}+\cdots+\right.$ $\left.e^{-T s}\right)\left(-E_{f}(s)\right)$

where the sampling rate $T$ is sufficient and $n$ is a sufficiently large number. It is reasonable to assume that

$\sum_{n=1}^{\infty} e^{-n T s} \cong(1-T s) / T s$.

The steady transfer function can be rewritten as:

$G(s)=\frac{\hat{E}(s)}{E_{f}(s)}=\frac{(\gamma \beta(1-\beta)(1-\sigma) T s+\gamma \beta(1-\beta) \sigma}{m_{d}(1-\beta) T s^{3}+b_{d} T s^{2}}$

The force error transfer function $\Phi(s)$ for the whole closedloop system is shown below:

$$
\begin{aligned}
& \Phi(s)=\frac{E(S)}{R(s)}=\frac{1}{1+G(s) H(s)} \\
& =\frac{m_{d}(1-\beta) T s^{3}+b_{d} T s^{2}}{m_{d}(1-\beta) T s^{3}+b_{d} T s^{2}+\left(\gamma \beta(1-\beta)(1-\sigma) k_{e} T s+\gamma \beta(1-\beta) \sigma\right.}
\end{aligned}
$$

Consider a complex dynamic environmental situation: a sine signal $r(t)=\sin \omega t$. Long division method is used to compute dynamic error of FO-AIC (taking the first three terms):

$$
\begin{aligned}
& e_{S S}(t)=\Phi(0) r(t)+\dot{\Phi}(0) \dot{r}(t)+\left(\frac{1}{2 !}\right) \ddot{\Phi}(0) \ddot{r}(t)+\cdots= \\
& -\omega^{2} \frac{2 b T}{\gamma \beta(1-\beta) k_{e}{ }^{2} \sigma} \sin \omega t+\cdots
\end{aligned}
$$

It can be seen that, for a complex environment, the tracking error can be decreased by increasing the update rate $\sigma$.

For transient response analysis, since the collision time is short, $\mathrm{n}$ is no longer being an infinite amount. Hence, rewriting the compensation part of Eq. (10) and dividing both sides by the sampling time, we obtain:

$\frac{\rho(t)-\rho(t-T)}{T}=\frac{\sigma \gamma \beta(1-\beta)}{b_{d}}\left(\frac{f_{d}(t-T)-f_{e}(t-T)}{T}\right)$

In an extremely small period, it can be approximated that $c(t-T) \cong c(t)=-e_{f}$. The function representation between $\rho(t)$ and $c(t)$ can be written as follows:

$\rho(t)=-\frac{\sigma \gamma \beta(1-\beta)}{b_{d}} e_{f}(t)$

Substituting Eq. (18) into Eq. (9) and then performing a Laplace transformation, the transient response transfer function of the impedance controller is obtained as the following:

$G(s)=\frac{\hat{E}(s)}{E_{f}(s)}=\frac{\gamma \beta(1-\beta)(1+\sigma)}{(1-\beta) m s^{2}+b s}$

The transient response transfer function $\Psi$ (s) for the whole closed-loop system is:

$\Psi(s)=\frac{G(s) H(s)}{1+G(s) H(s)}=\frac{\gamma \beta(1-\beta)(1+\sigma) k_{e}}{(1-\beta) m s^{2}+b s+\gamma \beta(1-\beta)(1+\sigma) k_{e}}$

The damping coefficient $\zeta$ about the oscillation form of the system can be calculated from Eq. (20) as:

$\zeta=\frac{b}{2(1-\beta) \sqrt{m \gamma \beta(1+\sigma) k_{e}}}$

In Eq. (21), it can be clearly seen that the system will present oscillation when $\sigma$ increases, which indicates that the smaller $\sigma$ having a better force overshoots the suppression effect.

Combining the transient response and steady-state tracking analysis, in general, keeping the $\sigma$ unchanged is an inadvisable 
choice. Therefore, a dynamic adaptive update rate based on fuzzy logic coupling into FO-AIC is introduced in the forthcoming section.

\subsubsection{Fuzzy logic controller}

Supposing $\left[-X_{e f}, X_{e f}\right],\left[-X_{\dot{e f}}, X_{\dot{e} f}\right]$ and $\left[-Y_{\sigma}, Y_{\sigma}\right]$ are the basic domains of $\mathrm{e}_{\mathrm{f}}, \dot{\mathrm{e}}_{\mathrm{f}}$ and $\sigma$, respectively, in this study, we set number 10 as the boundary for simplify. Based on the comparison between the effects of different types of membership functions on fuzzy controller performance, seven triangular type membership functions are chosen to demonstrate input and output of the proposed controller, as illustrated in Fig. 8. Seven linguistic values are described, namely positive big (PB), positive medium (PM), positive small (PS), zero (ZO), negative big (NB), negative medium (NM) and negative small (NS). The triangular membership function is used here mainly considering the computation speed. Similarly, the membership functions of the selected input and output are kept the same for the sake of calculation.

The fuzzy control rules generalise the relationships between the inputs and outputs. They are established based on the experiences and intuitions of the skilled workers. Both the error $\mathrm{e}_{\mathrm{f}}$ and the error ratio $\dot{e}_{f}$ have seven fuzzy subsets. Therefore, 49 fuzzy rules can be obtained according to the expertise, which are shown as Tab. 1.

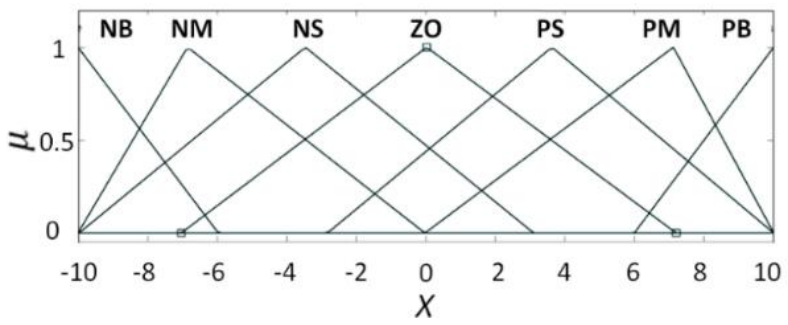

Fig. 8. Membership function of $e_{f}, \dot{e}_{f}$ and $\sigma$

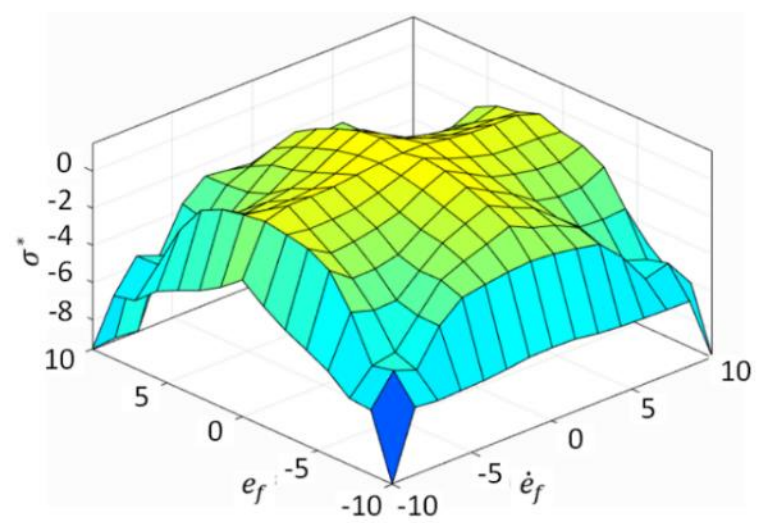

Fig. 9. Output curve-surface of fuzzy logic control

Tab. 1 can be applied as: if $e_{f}(i)=A_{j}$ and $\dot{e_{f}}(i)=B_{j}$, then $\sigma=C_{j}(j=1, \ldots, 49)$ and $A_{j}$ and $B_{j}$ are the fuzzy sets corresponding to $e_{f}(i)$ and $\dot{e_{f}}(i)$ in the j-th fuzzy rule, respectively. $C_{j}$ is the fuzzy outputs corresponding to $\sigma$ in the $j$-th fuzzy logical rule. The output membership grades for different fuzzy sets are derived from the rule table using the Mamdani fuzzy reasoning method, as seen in Fig. 9. The centre of gravity defuzzification method is selected to defuzzify the output fuzzy set:

$\sigma^{*}=\frac{\sum_{j=1}^{n} A_{j}\left(e_{f}\right) B_{j}\left(e_{f}\right) \mu_{i}}{\sum_{j=1}^{n} A_{j}\left(e_{f}\right) B_{j}\left(e_{f}\right)}$

Tab. 1. Fuzzy Control Logic Rule Table

\begin{tabular}{|l|l|l|l|l|l|l|l|l|}
\hline & & \multicolumn{7}{|c|}{$\dot{\boldsymbol{e}}_{\boldsymbol{f}}$} \\
\cline { 3 - 9 } & & NB & NM & NS & ZO & PS & PM & PB \\
\hline & NB & NB & NB & NM & PM & NM & NB & NB \\
\hline & NM & NB & NB & NM & PM & NM & NB & NB \\
\hline & NS & NM & NM & NS & PS & NS & NM & NM \\
\hline $\mathbf{e}_{\boldsymbol{f}}$ & ZO & NM & ZO & PM & PB & PM & ZO & NM \\
\hline & PS & NM & NM & NS & PS & NS & NM & NM \\
\hline & PM & NB & NB & NM & PM & NM & NB & NB \\
\hline & PB & NB & NB & NM & PM & NM & NB & NB \\
\hline
\end{tabular}

NB, negative big; NM, negative medium; NS, negative small; PB, positive big; PM, positive medium; PS, positive small; ZO, zero.

In Tab. $1, n$ is the fuzzy rule number, and $\mu_{j}$ is the membership grade of the $j$-th fuzzy output, which takes values in $[0,1]$. Suppose $Q_{\sigma}$ is the quantification factors of $\sigma$ undergoing conversion from the fuzzy domain to the basic domain, then $Q_{\sigma}$ can be given by:

$Q_{\sigma}=\frac{B_{\sigma}}{10}$

Then, the precise values of $\sigma$ can be denoted as:

$\sigma=Q_{\sigma} \sigma^{*}$

\subsubsection{Sigma boundary and stability}

To determine the $\sigma$ boundary $B_{\sigma}$, a stability analysis is carried out in this section. Then, stability analysis is given according to the Routh criterion.

Substituting Eq. (10) into Eq. (9) yields:

$e_{f}(t)=\frac{m_{d}}{\gamma \beta} \ddot{\hat{e}}(t)+\frac{b_{d}}{\gamma \beta(1-\beta)} \dot{\hat{e}}(t)+\frac{b_{d}}{\gamma \beta(1-\beta)} \rho(t-T)+$ $\sigma\left(f_{d}(t-T)-f_{e}(t-T)\right)$

Substituting the estimated position error $\hat{e}(t)=e(t)+$ $\delta p_{e}(t)$, Eq. (25) is rewritten as:

$e_{f}(t)=\frac{m_{d}}{\gamma \beta}\left[\ddot{e}(t)+\delta \ddot{p}_{e}(t)\right]+\frac{b_{d}}{\gamma \beta(1-\beta)}\left[\dot{e}(t)+\delta \dot{p}_{e}(t)\right]$

$+\frac{b_{d}}{\gamma \beta(1-\beta)} \rho(t-T)+\sigma\left(f_{d}(t-T)-f_{e}(t-T)\right)$

Reorganising Eq. (26) yields:

$\frac{m_{d}}{\gamma \beta} \ddot{e}(t)+\frac{b_{d}}{\gamma \beta(1-\beta)} \dot{e}(t)-e_{f}(t)+\frac{b_{d}}{\gamma \beta(1-\beta)} \rho(t-T)$

$+\sigma\left(f_{d}(t-T)-f_{e}(t-T)\right)=-\frac{m_{d}}{\gamma \beta} \delta \ddot{p}_{e}(t)-$

$\frac{b_{d}}{\gamma \beta(1-\beta)} \delta \dot{p}_{e}(t)$

According to the stiffness model between the robot and the environment, which is $f_{e}=k_{e}\left(p_{e}-p_{d}\right)=-k_{e} e$, after the 
differential it becomes:

$\dot{e}=-\dot{f}_{e} / k_{e}, \ddot{e}=-\ddot{f}_{e} / k_{e}$

Substituting Eq. (28) into Eq. (27) yields:

$-\frac{m_{d}}{\gamma \beta} \ddot{f}_{e}(t)-\frac{b_{d}}{\gamma \beta(1-\beta)} \dot{f}_{e}(t)-k_{e} e_{f}(t)$

$+\frac{b_{d}}{\gamma \beta(1-\beta)} k_{e} \rho(t-T)+\sigma k_{e}\left(f_{d}(t-T)-f_{e}(t-T)\right.$

$=-k_{e}\left(\frac{m_{d}}{\gamma \beta} \delta \ddot{p}_{e}(t)+\frac{b_{d}}{\gamma \beta(1-\beta)} \delta \dot{p}_{e}(t)\right)$

Let $\hat{f}_{e}(t)=k_{e} \delta p_{e}(t)$; then, adding the term $\frac{m_{d}}{\gamma \beta} \ddot{f}_{d}(t)+$ $\frac{b_{d}}{\gamma \beta(1-\beta)} \dot{f}_{d}(t)$ at both sides, Eq. (29) could be represented as:

$\frac{m_{d}}{\gamma \beta}\left(\ddot{f}_{d}(t)-\ddot{f}_{e}(t)\right)+\frac{b_{d}}{\gamma \beta(1-\beta)}\left(\dot{f}_{d}(t)-\dot{f}_{e}\right)-k_{e} e_{f}(t)+$ $\frac{b_{d}}{\gamma \beta(1-\beta)} k_{e} \rho(t-T)+\sigma k_{e}\left(f_{d}(t-T)-f_{e}(t-T)\right)=$

$\frac{m_{d}}{\gamma \beta}\left(\ddot{f}_{d}(t)-\ddot{\hat{f}}_{e}(t)\right)+\frac{b_{d}}{\gamma \beta(1-\beta)}\left(\dot{f}_{d}(t)-\dot{\hat{f}}_{e}(t)\right)$

Marking $c(t)=f_{d}(t)-f_{e}(t)$ and $r(t)=f_{d}(t)-\hat{f}_{e}(t)$, Eq. (30) can be simplified as follows:

$\frac{m_{d}}{\gamma \beta} \ddot{c}+\frac{b_{d}}{\gamma \beta(1-\beta)} \dot{c}+\frac{b_{d}}{\gamma \beta(1-\beta)} k_{e} \rho(t-T)+\sigma k_{e} c(t-T)+$

$k_{e} c=\frac{m_{d}}{\gamma \beta} \ddot{r}+\frac{b_{d}}{\gamma \beta(1-\beta)} \dot{r}$

Combining Eq. (11) and Eq. (31) yields:

$\frac{m_{d}}{\gamma \beta} \ddot{c}+\frac{b_{d}}{\gamma \beta(1-\beta)} \dot{c}+\sigma k_{e}(c(t-(n+1) T)+\cdots+$

$c(t-T))+k_{e} c=\frac{m_{d}}{\gamma \beta} \ddot{r}+\frac{b_{d}}{\gamma \beta(1-\beta)} \dot{r}$

Laplace transform of Eq. (32) is:

$\begin{aligned} \frac{c(s)}{r(s)}= & \frac{(1-\beta) m_{d} s^{2}+b_{d} s}{(1-\beta) m_{d} s^{2}+b_{d} s+k_{e} \gamma \beta(1-\beta)} \\ & +\sigma k_{e} \gamma \beta(1-\beta)\left(e^{\left.-(n+1) T s_{+} \cdots+e^{-T s}\right)}\right.\end{aligned}$

The stability of Eq. (33) can be guaranteed by the characteristic expressed as:

$(1-\beta) m_{d} s^{2}+b_{d} s+k_{e} \gamma \beta(1-\beta)+\sigma k_{e}\left(e^{-(n+1) T s}+\right.$ $\left.\cdots+e^{-T s}\right)=0$

Assuming that $\mathrm{n}$ is a sufficiently large number, and that the sampling rate $T$ is sufficient for $\sum_{n=1}^{\infty} e^{-n T s} \cong(1-T s) / T s$, we substitute it into Eq. (34) to obtain:

$(1-\beta) m_{d} T s^{3}+b_{d} T s^{2}+k_{e} T(\gamma \beta(1-\beta)-\sigma) s+$ $\sigma k_{e}=0$

According to the Routh criterion, the Routh array is presented as:

$$
\begin{array}{lll}
s^{3} & (1-\beta) m_{d} T & k_{e} T(\gamma \beta(1-\beta)-\sigma) \\
s^{2} & b_{d} T & \sigma k_{e} \\
s^{1} & \frac{b_{d} T^{2} k_{e}(\gamma \beta(1-\beta)-\sigma)-(1-\beta) m_{d} T \sigma k_{e}}{b_{d} T} & 0 \\
& \multicolumn{2}{c}{0}
\end{array}
$$

To ensure the stability of the system, the coefficients of the first column and the coefficients of the characteristic equation must be positive, which is represented as:

$$
\left\{\begin{array}{c}
\frac{b_{d} T^{2}(\gamma \beta(1-\beta)-\sigma)-(1-\beta) m_{d} T \sigma k_{e}}{b_{d} T}>0 \\
k_{e} T(\gamma \beta(1-\beta)-\sigma)>0 \\
\sigma k_{e}>0
\end{array}\right.
$$

Simplifying Eq. (37), the boundary of $\sigma$ is:

$0<\sigma<\frac{b_{d} T \gamma \beta(1-\beta)}{(1-\beta) m_{d}+b_{d} T}$

For a stable system, the steady-state error $e_{s s}$ can be defined based on the Laplace transform. For convergence, the steadystate error can be calculated as:

$$
\begin{aligned}
& e_{s s}=\lim _{s \rightarrow 0} s E(s)= \\
& \lim _{s \rightarrow 0} s\left[\frac{(1-\beta) m_{d} T s^{2}+b_{d} T s}{(1-\beta) m_{d} T s^{3}+b_{d} T s^{2}+k_{e} T(\gamma \beta(1-\beta)-\sigma) s}-1\right] r(s) \\
& +\sigma k_{e}
\end{aligned}
$$

When the input is a step function with the form as $r(s)=$ $1 / s$, Eq. (39) yields a result as follows:

$e_{s s}=\lim _{s \rightarrow 0} s(c(s)-r(s))=-1$

The following conclusion can be reached from Eq. (40):

$\lim _{s \rightarrow 0} s c(s)=0, \lim _{t \rightarrow \infty} c(t)=0$

Therefore, when $t \rightarrow \infty, f_{e} \rightarrow f_{d}$. The contact force converges to the desired force. Actually, even if $r(s)$ is a slope or sine input as in simulations and experiments, the force tracking error tends to zero, and this also can be proven.

\subsection{Control effect analysis}

For position-based impedance control systems, hierarchical separation of position control and impedance control is a common structure nowadays. This means that the stability of the whole system can be divided into two subsystems: impedance control and position control. It is a reasonable assumption that the position controller can achieve stable tracking in industrial robots. Therefore, we mainly study the stability of the impedance control part. The stability of the impedance control part is mainly composed of the following parts: pre-PID regulator, fuzzy controller and FO-AIC. Similarly, the performance of the controller is related to these three parts, which can be seen in Tab. 2. (The table is obtained by simulation analysis, based on the selection variable method.)

Tab. 2. The Control effect of pre-PID regulator, Fuzzy logic and FO-AIC

\begin{tabular}{|c|c|c|c|c|}
\hline \multicolumn{2}{|c|}{$\begin{array}{c}\text { Index } \\
\text { Controller }\end{array}$} & $\begin{array}{c}\text { Force } \\
\text { response } \\
\text { (Tracking } \\
\text { time) }\end{array}$ & $\begin{array}{c}\text { Force } \\
\text { overshoot } \\
\text { (Collision } \\
\text { force) }\end{array}$ & $\begin{array}{c}\text { Force } \\
\text { tracking } \\
\text { (Tracking } \\
\text { error) }\end{array}$ \\
\hline Pre-PID & $k_{p}(\uparrow)$ & High $(\downarrow)$ & High $(\uparrow)$ & Low $(\downarrow)$ \\
\hline $\begin{array}{c}\text { Fuzzy } \\
\text { logic }\end{array}$ & $\sigma(\uparrow)$ & Low $(\downarrow)$ & High $(\uparrow)$ & High $(\downarrow)$ \\
\hline FO-AIC & $\alpha$ or $\beta(\downarrow)$ & Low $(\uparrow)$ & High $(\downarrow)$ & Low $(\uparrow)$ \\
\hline
\end{tabular}

FO-AIC, fractional order-adaptive impedance control.

As can be seen from Tab. 2, each part has its own emphasis and strengths on the performance indicators of force control. The pre-PID can quickly improve the response speed and ensure the tracking accuracy, but at the same time will introduce a large 
overshoot. FO tools can greatly reduce overshoot of force control, but it is insufficient in response time and tracking accuracy. The $\sigma$ can greatly affect the overshoot and tracking accuracy, and thus fuzzy logic is needed to determine dynamic adjustment based on the contact information. Therefore, in order to obtain a force controller with fast response, small overshoot and high tracking accuracy, all three parts are indispensable.

\section{SIMULATIONS}

To verify the theoretical findings and controller performance, a series of simulation studies are conducted and presented in this section. To test the performance and adaptability of various strategies in a dynamic continuous stiffness environment, the stiffness is designed as:

$k_{e}=4000+800 \sin \left(\frac{\pi}{2} t\right)$

The motion process of the robot is as follows: first contacting the dynamic, uncertain surface to achieve a desired contact force, and then tracking the desired force.

\subsection{FO order impacts simulation}
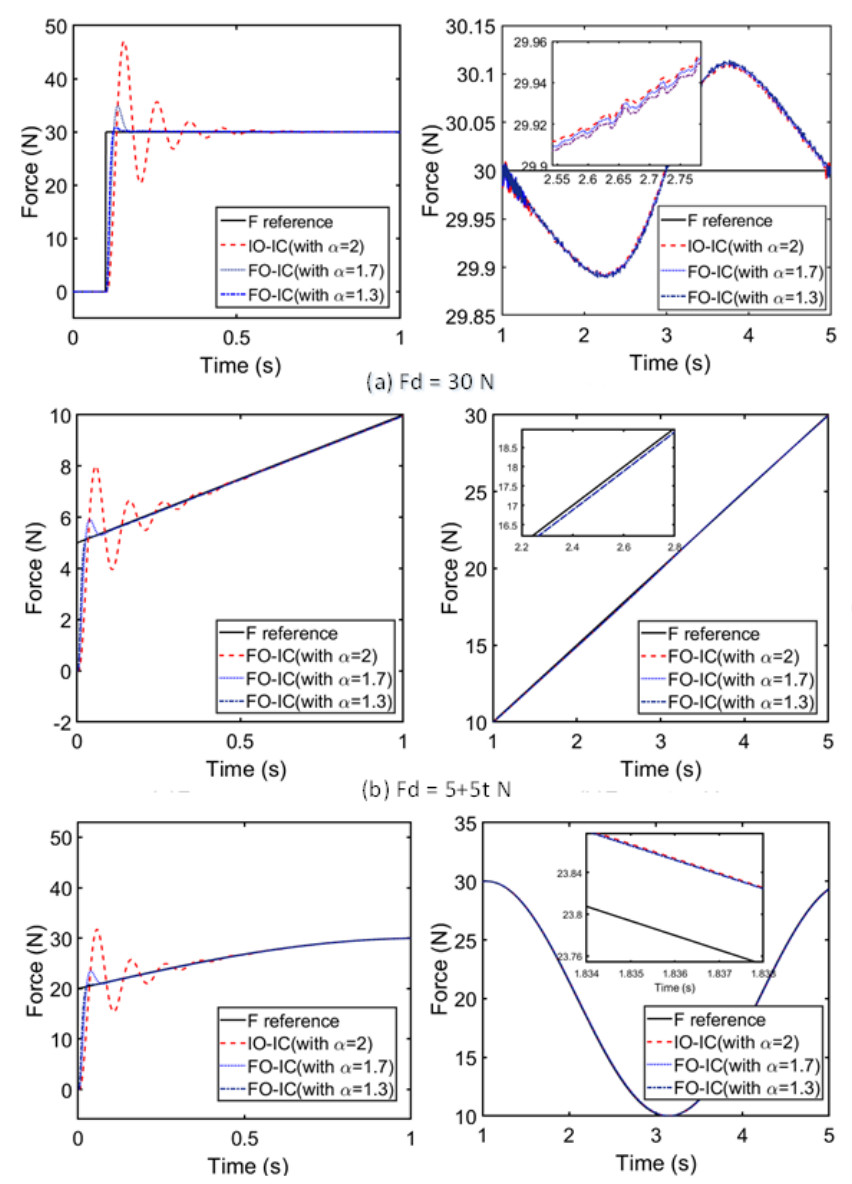

(c) $\mathrm{Fd}=10 * \sin 1.5 \mathrm{t}+20 \mathrm{~N}$

Fig. 10. Performance comparison of FO-AIC and IO-AIC for (a) constant force; (b) slope force; (c) sine force with dynamic stiffness. FO-AIC, fractional order-adaptive impedance control; IO-AIC, integer order adaptive impedance controller
Fig. 10 shows the simulation results of FO-AIC and IO-AIC for a constant force, a slope force and a sine force, respectively. $0 \sim 1 s$ is the initial contact state, $1 \sim 5 s$ are the tracking state. As can be seen in the contact stage, as the order decreases, the force overshoots also decrease, which indicates that the smaller the order, the smaller the collision force. Meanwhile, we can see that the force tracking has a slight improvement in the tracking stage, but not much. Therefore, the proposed FO-IC can greatly reduce force overshoots in the initial contact phase compared with IO-IC, and this effect has strong adaptability and robustness for various tasks.

\subsection{1-DOF robot contact force tracking simulation}

The basic impedance parameters $m_{d}=1 \mathrm{Ns} \mathrm{s}^{2} / \mathrm{m}$ and $b_{d}=44 \mathrm{Ns} / \mathrm{m}$ are selected based on experiences (here referring to the benchmark). Modelling uncertainty is introduced in simulation implementations by considering the estimated mass $\widehat{m}_{r}=0.8 \mathrm{~kg}$ instead of the real mass $m_{r}=1 \mathrm{~kg}$. The position controller is designed with high gains to achieve good position tracking, which is common in practice. The unmoulded friction $\mathrm{F}_{\mathrm{f}}$ is assumed to have a form, with $c_{v}=1.2 \mathrm{Ns} / \mathrm{m}$ and $F_{c}=4 \mathrm{~N}$ as the coefficients of viscous and coulomb friction:

$$
F_{f}=-\operatorname{sign}(\dot{x})\left(c_{v}|\dot{x}|+F_{c}\right)
$$
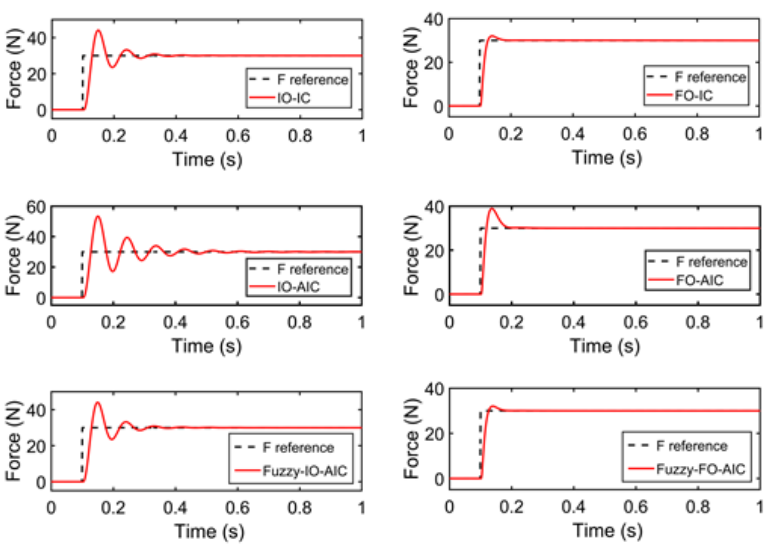

(a) Force response
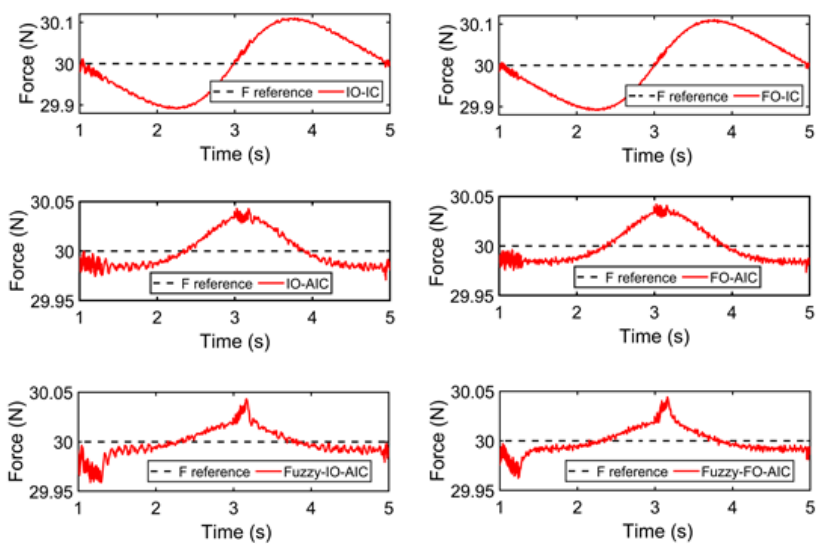

(b) Force tracking

Fig. 11. Constant force control performance comparison of Fuzzy-FOAIC with other controllers. Fuzzy-FO-AIC, fuzzy FO adaptive impedance control

Figs. 11 and 12 show the force response, overshoots and tracking performance of the classical control methods (IO-IC [7], 


\section{sciendo}

Hongli Cao

IO-AIC [12]), Fuzzy-IO-AIC [19], contrast controller (FO-IC [30], FO-AIC [21]) and the proposed Fuzzy-FO-AIC control strategies in different scenarios. (The FOs are all replaced by the FO with IOA.)

First, the transient response of the step and sine force are shown in Fig. 11 (a) and Fig. 12 (a) with a time range of 0-1 s, respectively. The contact force has a stronger vibration and force overshoots in IO-AIC at the initial contact stage, followed by Fuzzy-IO-AIC, IO-AIC and FO-AIC. However, both FO-IC and Fuzzy-FO-AIC have a superior force overshoots suppression ability. Lateral results' comparison show that FO tools have better vibration suppression than $\mathrm{IO}$ due to their natural damping effect. Longitudinal results' comparison shows that a smaller $\sigma$ has a smaller force overshoot. Taken together, the Fuzzy-FO-AIC actually achieve the double damping effect, which indicates that this approach has the best force overshoots suppression ability.
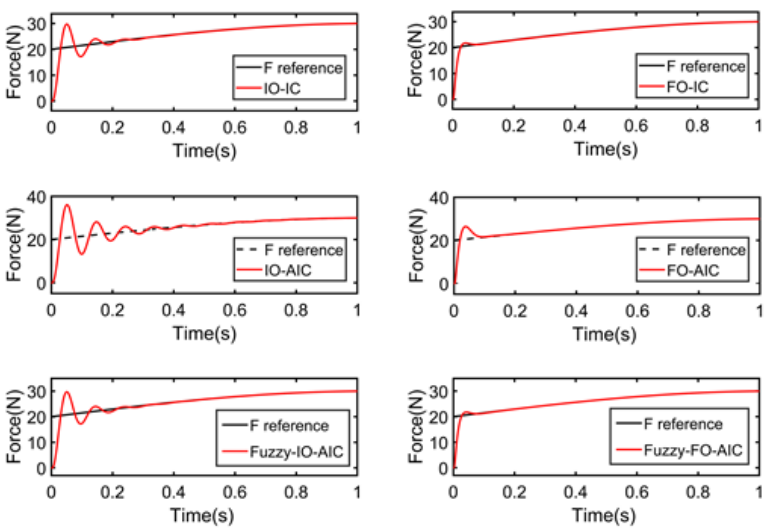

(a) Force response
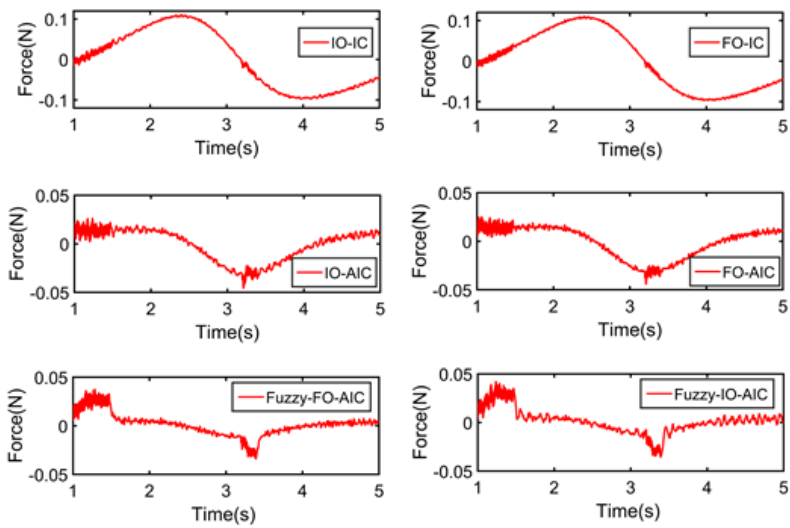

(b) Force tracking error

Fig. 12. Varying force control performance comparison of Fuzzy-FO-AIC with other controllers. Fuzzy-FO-AIC, fuzzy FO adaptive impedance control

Then, the corresponding steady force tracking performance is highlighted in Fig. 11(b) and Fig. 12(b) with a time ranging from $1 \mathrm{~s}$ to $5 \mathrm{~s}$. Unlike the vibration collision phase, IO-IC and FO-IC have the worst tracking effect of all. IO-AIC, FO-AIC and their adaptive versions Fuzzy-IO-AIC, Fuzzy-FO-AIC have the best tracking effects since all their $\sigma$ ups to the upper bound. Therefore, it can be concluded that the force tracking accuracy of either FO or IO depends only on the value of $\sigma$, the larger $\sigma$ and the higher precision. In theory, the Fuzzy-IO-AIC and Fuzzy-FO-AIC controllers all can achieve more accurate force tracking effects owing to the fact that the $\sigma$ boundary can be enlarged by the prePID regulator. (a)

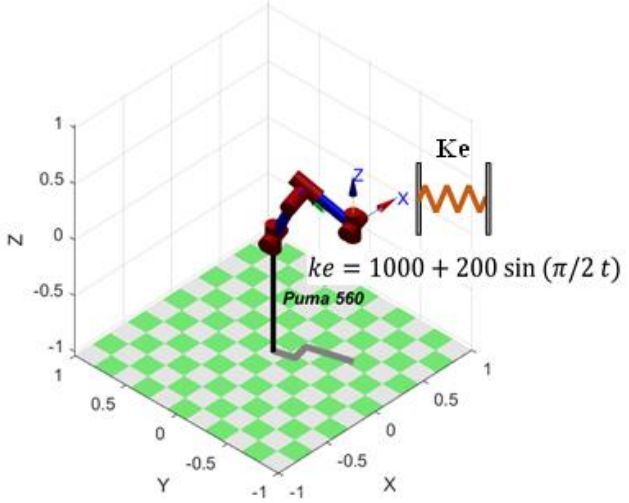

(b)

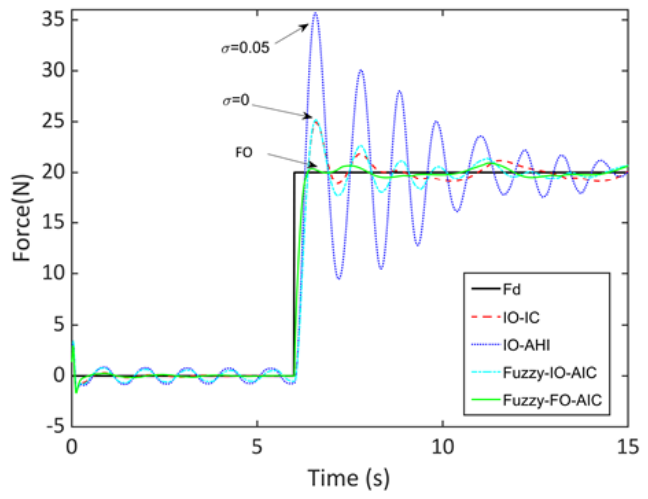

(c)

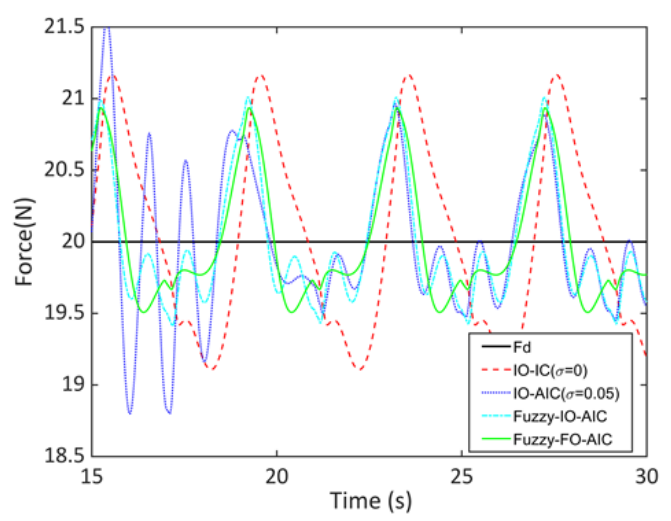

Fig. 13. 6-DOF robot force control grinding simulation

From the simulation results and analysis, we observe that introducing fuzzy adaptive $\sigma$ can effectively balance the impact force and tracking requirements. Meanwhile, FO is used to further enhance control system stability. Hence, the Fuzzy-IO-AIC control strategy is the best choice of all to achieve a superior force controller, namely one with the characteristics of high force tracking and no overshoots.

\subsection{6-DOF robot dynamic contact simulation}

The Fuzzy-FO-AIC is arranged in the form of a 6-DOF robot (PUMA560) for the polishing simulation study, with force control in the $x$-direction of the motion constraint, and motion control in the remaining directions, as indicated in Fig. 13(a). Basic cartesian impedance parameters are set: $m_{d}=30 \mathrm{Ns}^{2} / \mathrm{m}$ and $b_{d}=$ $120 \mathrm{Ns} / \mathrm{m}$; and the approximation coefficient of $\mathrm{FO}$ is $\beta=0.7$. The robot is controlled by inverse dynamics plus joint servo for motion tracking, with PD parameters $\mathrm{k}_{\mathrm{p}}=1 \mathrm{e}^{3}$ and $k_{d}=$ 


\section{The sampling period is $4 \mathrm{~ms}$.}

Fig. 13(b) shows the IO-IC, IO-AIC, Fuzzy-IO-AIC and FuzzyFO-AIC in the contact phase. It can be seen that IO-AIC also exhibits a large overshoot behaviour, and as the update rate decreases, the vibration overshoot has a great reduction in IO-IC and Fuzzy-IO-AIC, but does not disappear completely. In contrast, there is almost no overshoot in Fuzzy-FO-AIC. As can be seen from the steady-state tracking phase in Fig. 13(c), the IO-IC tracking accuracy is poor at about $10.5 \%$, while the IO-AIC, Fuzzy-IOAIC and Fuzzy-FO-AIC tracking accuracy improves at about $7 \%$. The Fuzzy-FO-AIC demonstrates a transient and steady-state control performance that is much better than the current commonly used impedance control strategies. Essentially, fractional-order impedance can further improve the tracking accuracy to a certain extent by appropriately increasing the update rate due to its better stability.

\section{CONCLUSIONS}

The significance of the robot contact operation has been growing recently due to the introduction of interactive robots. Maintaining the high speed, stable, smooth contact and high precision force tracking indicators are some of the highly challenging aspects involved in force controller design. Additionally, these demands are among the most common issues in real robot application.

In this paper, the Fuzzy-FO-AIC is proposed to manage dynamic contact force tracking in an uncertain environment (e.g., polishing tasks). The need to adjust the dynamic update rate and maintain the superiority of FO control are highlighted. A fuzzy logic controller inferring the update rate on-line is applied and the stability and boundary are analysed. Meanwhile, an 10 approximate method using percentage to simplify fractional control is also provided and verified through simulation. The simulation results all show that Fuzzy-FO-AIC can improve the dynamic force tracking performance, significantly in an uncertain environment, better than other previous controllers. In particular, FO control enables more effective control and provides more flexibility to adjust stability than 10, making it well suited for robotic applications involving force control. Furthermore, the reported methodology can be expanded to force control in other tasks where an unknown environment is easily distorted or manufacturing requires force control based on industrial robots.

\section{REFERENCES}

1. Liang L, Chen Y, Liao L, Sun H, Liu YJR, Manufacturing C-I. A novel impedance control method of rubber unstacking robot dealing with unpredictable and time-variable adhesion force. 2021;67:102038.

2. Cao H, He Y, Chen X, Zhao XJIRtijorr, application. Smooth adaptive hybrid impedance control for robotic contact force tracking in dynamic environments. 2020.

3. Mokhtari M, Taghizadeh M, Mazare MJR. Hybrid adaptive robust control based on CPG and ZMP for a lower limb exoskeleton. 2021;39(2):181-99.

4. Dong Y, Ren T, Wu D, Chen KJJol, Systems R. Compliance control for robot manipulation in contact with a varied environment based on a new joint torque controller. 2020;99(1):79-90.
5. Raibert MH, Craig JJ. Hybrid position/force control of manipulators. 1981.

6. Mason MTJIToS, Man,, Cybernetics. Compliance and force control for computer controlled manipulators. 1981;11(6):418 32.

7. Hogan N. Impedance control: An approach to manipulation: Part l-Theory. 1985.

8. Komati B, Pac MR, Ranatunga I, Clévy C, Popa DO, Lutz P, editors. Explicit force control vs impedance control for micromanipulation. International Design Engineering Technical Conferences and Computers and Information in Engineering Conference; 2013: American Society of Mechanical Engineers.

9. Wu J, Ni F, Zhang Y, Fan S, Zhang Q, Lu J, et al. Smooth transition adaptive hybrid impedance control for connector assembly. 2018.

10. Akdoğan E, Aktan ME, Koru AT, Arslan MS, Atlıhan M, Kuran BJM. Hybrid impedance control of a robot manipulator for wrist and forearm rehabilitation: Performance analysis and clinical results. 2018;49:77-91.

11. Jung S, Hsia TC, Bonitz RGJIToCST. Force tracking impedance control of robot manipulators under unknown environment. 2004;12(3):474-83.

12. Duan J, Gan Y, Chen M, Dai XJR, Systems A. Adaptive variable impedance control for dynamic contact force tracking in uncertain environment. 2018;102:54-65.

13. Solanes JE, Gracia L, Muñoz-Benavent P, Esparza A, Miro JV, Tornero JJR, et al. Adaptive robust control and admittance control for contact-driven robotic surface conditioning. 2018;54:115-32.

14. Lu Z, Goldenberg AAJTljorr. Robust impedance control and force regulation: Theory and experiments. 1995;14(3):225-54.

15. Fateh MM, Khorashadizadeh SJND. Robust control of electrically driven robots by adaptive fuzzy estimation of uncertainty. 2012;69(3):1465-77.

16. Li Y, Ge SS, Zhang Q, Lee THJICT, Applications. Neural networks impedance control of robots interacting with environments. 2013;7(11):1509-19.

17. Cao H, Chen X, He Y, Zhao XJIA. Dynamic adaptive hybrid impedance control for dynamic contact force tracking in uncertain environments. 2019;7:83162-74.

18. Xu WJJoDS, Measurement,, Control. Robotic time-varying force tracking in position-based impedance control. 2016;138(9):091008.

19. Sheng $X$, Zhang XJM. Fuzzy adaptive hybrid impedance control for mirror milling system. 2018;53:20-7.

20. Zhou Q, Li H, Shi PJIToFS. Decentralized adaptive fuzzy tracking control for robot finger dynamics. 2014;23(3):501-10.

21. Nikdel N, Badamchizadeh M, Azimirad V, Nazari MAJITolE. Fractional-order adaptive backstepping control of robotic manipulators in the presence of model uncertainties and external disturbances. 2016;63(10):6249-56.

22. Zhong J, Li LJltocst. Tuning Fractional-Order $\$\{P \mid\}^{\wedge}\left\{\right.$ llambda\} $\{D\}^{\wedge}\{\mid \mathrm{mu}\}$ \$ Controllers for a Solid-Core Magnetic Bearing System. 2015;23(4):1648-56.

23. Padula F, Visioli AJICT, Applications. Optimal tuning rules for proportional-integral-derivative and fractional-order proportional-integral-derivative controllers for integral and unstable processes. 2012;6(6):776-86.

24. Aguila-Camacho N, Duarte-Mermoud MAJlt. Fractional adaptive control for an automatic voltage regulator. 2013;52(6):807-15. 
25. Shahri ESA, Alfi A, Machado JTJASC. Fractional fixedstructure $\mathrm{H}_{\infty}$ controller design using augmented lagrangian particle swarm optimization with fractional order velocity. 2019;77:688-95.

26. Haji VH, Monje CAJAsc. Fractional order fuzzy-PID control of a combined cycle power plant using Particle Swarm Optimization algorithm with an improved dynamic parameters selection. 2017;58:256-64.

27. Efe MÖJIToll. Fractional order systems in industrial automation-a survey. 2011;7(4):582-91.

28. Ahmed S, Wang H, Tian YJAJoC. Robust adaptive fractionalorder terminal sliding mode control for lower-limb exoskeleton. 2019;21(1):473-82.

29. Efe MÖJTotloM, Control. Integral sliding mode control of a quadrotor with fractional order reaching dynamics. 2011;33(8):985-1003.

30. Feliu-Talegon D, Feliu-Batlle V, Tejado I, Vinagre BM, HosseinNia SHJIt. Stable force control and contact transition of a single link flexible robot using a fractional-order controller. 2019;89:139-57.
31. Muñoz-Vázquez AJ, Gaxiola F, Martínez-Reyes F, ManzoMartínez AJAsc. A fuzzy fractional-order control of robotic manipulators with PID error manifolds. 2019;83:105646.

32. Oustaloup A, Levron F, Mathieu B, Nanot FMJIToC, Theory SIF, Applications. Frequency-band complex noninteger differentiator: characterization and synthesis. 2000;47(1):2539.

33. Wang Y, Luo G, Gu L, Li XJJoV, Control. Fractional-order nonsingular terminal sliding mode control of hydraulic manipulators using time delay estimation. 2016;22(19):39984011.

Acknowledgement: This work was supported by the National Key R\&D Program of China (2017YFB1301400).

Hongli Cao: (iD) https://orcid.org/0000-0003-4324-3785 